\title{
PANDEMIA: ENTRAVES IDENTIFICADOS NO PROCESSO DE ENSINO-APRENDIZAGEM DURANTE O USO DO ENSINO REMOTO EM ALUNOS DE GRADUAÇÃO DO MUNICÍPIO DE GUARULHOS'
}

\section{PANDEMIA: IDENTIFIED OBSTACLES IN THE TEACHING-LEARNING PROCESS DURING THE USE OF REMOTE TEACHING IN UNDERGRADUATE STUDENTS IN THE MUNICIPALITY OF GUARULHOS}

\author{
Alexandre Augusto Pollon ${ }^{2}$, Armando Rocha Júnior ${ }^{3}$, Tatiana Lima de Almeida ${ }^{4}$
}

Submetido em: 13/08/2021

Aprovado em: 17/09/2021

\section{RESUMO}

O presente projeto tem como tema central a investigação de aspectos provenientes do processo de ensino e aprendizagem durante a pandemia (COVID-19) alunos de graduação do município de Guarulhos, assim como, possíveis fatores emocionais envolvidos neste contexto. Pretende-se ainda, compreender de que maneira esta modalidade de ensino, dentro do contexto supracitado, está relacionada a alterações cotidianas e possíveis entraves psicossociais ou psicopatológicas. Para tanto, pretende-se realizar, aplicação das Escalas de Depressão (BDI-II), Ansiedade (BAI) e de Desesperança (BHS) de Beck, em alunos dos cursos de graduação do município de Guarulhos, dentro período letivo, em diferentes cursos. Os resultados serão apresentados oportunamente.

PALAVRAS-CHAVE: Covid-19. Depressão. Ansiedade. Desesperança. Estudantes universitários. Saúde mental.

\begin{abstract}
This project has as its central theme the investigation of aspects arising from the teaching and learning process during the pandemic (COVID-19) undergraduate students in the city of Guarulhos, as well as possible emotional factors involved in this context. It is also intended to understand how this teaching modality, within the aforementioned context, is related to daily changes and possible psychosocial or psychopathological barriers. Therefore, it is intended to carry out the application of Beck's Depression Scales (BDI-II), Anxiety (BAI) and Hopelessness (BHS) in undergraduate students in the city of Guarulhos, within the academic period, in different courses. The results will be presented in due course.
\end{abstract}

KEYWORDS: Covid-19. Depression. Anxiety. Hopelessness. College students. Mental health.

\footnotetext{
1 Trabalho Premiado na XVIII Jornada de Iniciação Científica da UNG- dezembro 2020 - Categoria Projeto de Pesquisa.

${ }^{2}$ Aluno do Curso de Graduação em Psicologia da Universidade Univeritas UNG, participante do Programa de Iniciação Científica PIC-UNG. e-mail: alexandre@pollon.com.br

${ }^{3}$ Dr. Prof. da Universidade Univeritas UNG Orientador. e-mail: armandopsico@uol.com.br

${ }^{4}$ Dra. Profa. da Universidade Univeritas UNG Orientador. e-mail: tati_tla@yahoo.com.br.
} 


\section{INTRODUÇÃO}

Assim que o novo coronavírus atingiu o primeiro país, a transmissão do COVID-19 se espalhava pelo mundo todo, trazendo consigo um cenário catastrófico e sem precedentes. COVID significa Corona Vírus Disease (Doença do Coronavírus), enquanto "19" se refere a 2019, segundo a Fundação Oswaldo Cruz (FIOCRUZ), a denominação é importante para evitar casos de xenofobia e preconceito, além de confusões com outras doenças.

Uma epidemia como esta, implica em uma perturbação psicossocial que pode ocorrer em diferentes níveis de intensidade e gravidade. As consequências ainda não estão mapeadas e existem pesquisas preliminares indicando para um possível grande número de casos acometidos por problemas em saúde mental em razão da incapacidade de enfrentamento da população afetada.

No Brasil, a fim de conter o avanço das transmissões, muitos estados e municípios decretaram leis de quarentena. Dessa forma, o impacto do isolamento social, diante da situação de pandemia, envolveu diversas dificuldades para a população em geral. Este projeto propõe a compreensão acerca das características e manifestações emocionais de alunos universitários de diferentes cursos de graduação do município de Guarulhos a partir desse cenário emergencial, ou seja, alunos em situação de imponderabilidade no seu processo de aprendizagem. Segundo Spink (2001), imponderabilidade é a hipótese de estarmos vivenciando a passagem de uma sociedade pautada pela noção de risco.

A pesquisa levantará as possíveis dificuldades encontradas pelos alunos de graduação, tais como a alteração da sua rotina cotidiana e possíveis alterações de funções cognitivas, dentre elas, o humor, a ansiedade e outros fatores psicopatológicos (DALGALARRONDO, 2000). De acordo Judith Beck (1997) eventos pós-traumáticos já existentes corroboram para o surgimento de sentimentos disfóricos e comportamentos desadaptativos, o que pode favorecer o desenvolvimento de patologias.

Apesar de os cursos de graduação a serem estudados neste trabalho oferecerem a modalidade de ensino presencial, em razão do momento extraordinário atual, as aulas passaram a ocorrer de maneira remota conforme autorização do Ministério da Educação e Cultura, Portaria Nº 343, de 17 de março de 2020 (BRASIL, 2020). O ensino remoto é diferente do Ensino à Distância $(E a D)$, pois, traz uma mudança temporária na entrega de instruções alternativas devido a circunstâncias de crise. Envolve o uso de soluções de ensino totalmente remotas para educação que, de outra forma, seriam ministradas presencialmente. Neste contexto, conta-se com a presencialidade virtual a partir de atividades síncronas.

Diante deste contexto, os alunos precisaram se adaptar de maneira rápida aos novos modelos do ensino remoto e, por esses fatores, assim como as dificuldades trazidas com ele no processo de adaptação, alguns foram influenciados na escolha sobre a permanência ou desistência da continuidade do curso.

Propõe-se investigar, a relação do impacto causado pelo isolamento social e o desempenho nos estudos em modalidade remota em alunos universitários nos cursos de graduação do município de Guarulhos. Para tanto, pretende-se usar como instrumentos de avaliação inventários psicológicos. Dessa maneira, pretende-se buscar informações sobre o perfil emocional do estudante de graduação em contexto emergencial da pandemia COVID-19.

Este trabalho pretende contribuir com a sociedade a fim elencar dados que ofereçam subsídios para identificação de estratégias de promoção da saúde e bem-estar dos alunos universitários, assim como, oferecer às universidades elementos que permitam auxiliar seus estudantes em seu processo de formação.

Na perspectiva acadêmica, o trabalho revela a sua relevância científica propondo oferecer subsídios técnicos a respeito das possíveis dificuldades de alunos em situação de catástrofe emergencial, auxiliando assim, na elaboração de novas pesquisas em saúde mental, processos de ensino e aprendizagem em situações de imponderabilidade. 


\section{OBJETIVOS}

Geral:

Investigar possíveis impactos do isolamento social e ensino remoto em situação de pandemia em alunos de graduação do município de Guarulhos.

Específicos:

- Verificar a presença de traços de ansiedade em alunos de todos os anos dos cursos de graduação do município de Guarulhos.

- Verificar a presença de traços depressivos em alunos de todos dos cursos de graduação do município de Guarulhos.

- Verificar a presença de traços desesperança em alunos de todos os anos dos cursos de graduação do município de Guarulhos.

- Identificar a presença tanto de traços depressivos, ansiosos e com desesperança concomitantes, em alunos do município de Guarulhos.

- Identificar a relação do ensino remoto emergencial e a presença de indicadores de ansiedade, depressão e desesperança.

- Subsidiar pesquisas tanto de cunho acadêmico quanto social em situações emergenciais, que busquem a promoção da saúde e bem-estar de estudantes.

- Oferecer mapeamento quantitativo sobre ansiedade, depressão e desesperança a respeito de alunos de graduação do município de Guarulhos.

\section{JUSTIFICATIVA}

A pesquisa será realizada com estudantes de graduação do município de Guarulhos voluntários. Este trabalho pretende contribuir com a sociedade a fim elencar dados que ofereçam subsídios para identificação de estratégias de promoção da saúde e bem-estar dos alunos universitários, assim como, oferecer às universidades elementos que permitam auxiliar seus estudantes em seu processo de formação. Na perspectiva acadêmica, o trabalho revela a sua relevância científica propondo oferecer subsídios técnicos a respeito das possíveis dificuldades de alunos em situação de catástrofe emergencial, auxiliando assim, na elaboração de novas pesquisas em saúde mental, processos de ensino e aprendizagem em situações de imponderabilidade.

A relevância acadêmica se mostra no fato de que os dados obtidos nos resultados poderão subsidiar novas pesquisas e aprofundar pesquisas já existentes acerca da condição emocional de estudantes universitários. Podendo ainda, de maneira preliminar, oferecer informações, para trabalhos acadêmicos que busquem identificar ou se empenhar no desenvolvimento de práticas de bons cuidados em relação à qualidade de vida destes estudantes.

A pesquisa será exclusivamente direcionada população de estudantes das Universidades de Guarulhos nos mais diversos cursos e disciplinas. O projeto de pesquisa está fundamentado em duas linhas de pesquisas convergentes, a Neurociência que estuda os processos cognitivos e o Desenvolvimento humano assim como os processos de adaptação ao meio. No campo da neurociência existem estudos a respeito das habilidades sócios emocionais que, diferem das habilidades sociais, por ser mais ampla e ter um olhar para o indivíduo também, não estando relacionado a apenas aspectos sociais. Estão sendo desenvolvidas pesquisas que apontam pessoas com habilidades socioemocionais mais estruturadas, possuem atitudes mais relevantes sobre si, sabendo lidar melhor com desafios, com mais perseverança, apresentando mais autoestima, autoeficácia, e até um maior comprometimento escolar/ acadêmico (DAMÁSIO, 2017). 


\section{REVISÃO BIBLIOGRÁFICA E/OU FUNDAMENTAÇÃO TEÓRICA}

O foco de estudo inicial está centrado em estudos acerca dos conceitos de ansiedade, depressão e desesperança, assim como os procedimentos de validação dos instrumentais a serem utilizados na coleta de dados.

\section{Ansiedade}

É um termo usado para vários distúrbios ou transtornos em geral. Todo indivíduo em algum momento da vida experimenta a sensação de ansiedade, seja por medo nervosismo, medo, apreensão e preocupação, os chamados transtornos de ansiedade incluem transtornos que compartilham características de e ansiedade excessivos e perturbações comportamentais relacionados.

\section{Depressão}

A depressão é uma doença psiquiátrica crônica e recorrente que produz alteração do humor caracterizada por tristeza profunda e forte sentimento de desesperança. É essencial identificar sintomas e procurar ajuda médica. A gravidade está baseada no número de sintomas dos critérios, em sua gravidade e no grau de incapacitação funcional.

Os casos considerados como "Leve" possuem as seguintes características: poucos os sintomas presentes além daqueles necessários para fazer o diagnóstico, a intensidade dos sintomas causa sofrimento, mas é manejável, e os sintomas resultam em pouco prejuízo no funcionamento social ou profissional. Já aqueles com classificação "Moderada" estão descritos da seguinte maneira: o número de sintomas, sua intensidade e/ou o prejuízo funcional estão entre aqueles especificados para "leve" e "grave". Os casos considerados Graves indicam que o número de sintomas está substancialmente além do requerido para fazer o diagnóstico, sua intensidade causa grave sofrimento e não é manejável, e os sintomas interferem acentuadamente no funcionamento social e profissional.

\section{Desesperança}

A principal característica da desesperança é influenciada pela visão negativa da realidade em cada contexto. O sentimento de desesperança causa nas pessoas um dor interior, traz a tristeza, baixa autoestima, solidão, esgotamento físico e cansaço mental. Entende-se que um estado de desesperança é aquele que pode ser ativado a qualquer momento, como, por exemplo, em um momento anterior à tentativa de suicídio. A desesperança pode ser distinguida entre seus status de estado e traço. O Brasil está entre os 10 países que registram os maiores números absolutos de suicídio, uma média de 24 mortes a cada dia. Do total de mortos, 79,3\% são homens, prevalecendo uma razão de 3,8 homens para cada mulher, de acordo com o Ministério da Saúde (BRASIL, 2007).

\section{Os inventários de ansiedade, depressão e desesperança}

Os inventários escolhidos para o levantamento de dados são o Inventário Beck de Depressão, Inventário Beck de Ansiedade e Inventário Beck de Desesperança configurados como testes psicológicos de uso exclusivo da Psicologia.

\section{BDI - II - Inventário Beck de Depressão - Segunda Edição}

As escalas de depressão de Beck visam avaliar a gravidade dos sintomas depressivos, sendo consideradas ferramentas importantes no diagnóstico, no prognóstico e na evolução da sintomatologia dos pacientes, bem como na uniformização da comunicação entre profissionais. A primeira versão do inventário (BDI-I) foi desenvolvida por 
Beck, Ward, Mendelson, Moch e Erbaugh (1961), sendo adaptada para a população brasileira por Cunha (2001). A segunda versão (BDI-II;) baseou-se na revisão da primeira edição com a finalidade de se alinhar aos critérios para os transtornos depressivos fornecidos pelo Manual Diagnóstico e Estatístico de Transtornos Mentais - Quarta Edição (DSM-IV; Associação Americana de Psiquiatria, 1995). Essa versão foi adaptada à população brasileira por Gorenstein, Pang, Argimon e Werlang (2011).

Em relação aos critérios de classificação em cada um dos Níveis de Depressão do BDI: Nível Mínimo de Depressão De 0 a 11 pontos; Nível Leve de Depressão De 12 a 19 pontos; Nível Moderado de Depressão De 20 a 35 pontos; Nível Grave de Depressão De 36 a 63 pontos.

Este instrumento não serve para fazer diagnóstico psiquiátrico por não envolver avaliação clínica. Entretanto, este inventário pode ser valioso para documentar a presença de sintomas depressivos e avaliar a sua gravidade. Tanto em indivíduos da população geral quanto nos pacientes deprimidos diagnosticados clinicamente (GORENSTEIN, 2011, p.56).

\section{BAI - Inventário Beck de Ansiedade}

O Inventário Beck de Ansiedade - BAI - (Beck Anxiety Inventory) (CUNHA, 2001) foi originalmente desenvolvido por Beck, Epstein, Brown e Steer, em 1988 e adaptado por Cunha (2001), apresentando bons coeficientes de fidedignidade e validade. Na primeira amostra estavam inclusos, idosos, universitários, reclusos de uma penitenciária, bombeiros, funcionários de hospitais e empresa de trens, adolescentes e estudantes de primeiro grau. Consiste em 21 descrições de sintomas de depressão com alternativas de 0 a 3 pontos, que devem ser assinaladas em razão da frequência de ocorrência dos mesmos.

Em relação aos critérios de classificação em cada um dos Níveis de Ansiedade do BAI os valores correspondentes a cada um é: Nível Mínimo de Ansiedade De 0 a 10 pontos; Nível Leve de Ansiedade De 11 a 19 pontos; Nível Moderado de Ansiedade De 20 a 30 pontos; Nível Grave de Ansiedade De 31 a 63 pontos. (CUNHA, 2001).

Conforme Beck e Steer (1993), o BAI foi construído para medir sintomas de ansiedade, que são compartiIhados de forma mínima com os de depressão. A sua versão em português foi utilizada em grupos psiquiátricos e não-psiquiátricos, inclusive em estudantes, e em trabalhos que envolvam outros sujeitos da comunidade (CUNHA, 2001, p.5).

\section{BHS - Inventário de Desesperança}

A Escala de Desesperança (BHS) compara a falta de esperança no futuro, que mediante a intensidade proporciona maior vulnerabilidade para o desenvolvimento de diversos transtornos mentais. A BHS é uma escala dicotômica com 20 itens com afirmações que envolvem cognições sobre a desesperança. A BHS é uma medida de pessimismo e oferece indícios sugestivos de risco de suicídio em sujeitos deprimidos ou que tenham história de tentativa de suicídio. Respostas: Certo $(C)$ e Errado (E). Tempo: forma autoadministrada: em torno de 5 a 10 minutos; Administração oral: aproximadamente 15 minutos; aplicado especialmente a pacientes psiquiátricos de 17 a 80 anos; Aplicação coletiva ou individual; O escore total é o resultado da soma dos itens individuais, cuja resposta aponta no crivo vale 1 ponto cada. Pode variar de 0 a 20, que é a estimativa da extensão das expectativas negativas frente ao futuro, classificadas em níveis; Normas brasileiras para pacientes psiquiátricos: Nível: Escore Mínimo 0 4, Leve 5-8, Moderado $9-13$ e Grave $14-20$. A BHS é um instrumento adequado como indicador psicométrico de risco de suicídio, mostrando-se mais útil em pacientes com histórico de depressão e de tentativa de suicídio. (CUNHA, 2001).

\section{MÉTODOS E PROCEDIMENTOS}

O trabalho proposto será iniciado com uma pesquisa teórica contínua sobre o tema proposto utilizando como 
palavras geradoras: ansiedade, depressão, desesperança, estudantes universitários, ensino remoto, emergência e pandemia.

Em um segundo momento será identificada a amostra de pessoas que se disponibilizarão a participar da pesquisa. A população está definida como alunos de graduação do município de Guarulhos em diferentes anos e instituições. A amostra será selecionada a partir de contato prévio por meio de redes sociais e as entrevistas agendadas de acordo com a melhor conveniência dos participantes e orientações técnicas do Conselho Regional de Psicologia no momento a serem executadas. Serão aplicadas as escalas BDI - II, BAI e BHS em cinquenta estudantes de graduação que estudem em instituições do ensino superior no município de Guarulhos. As aplicações ocorrerão de acordo com as normas e orientações do Conselho Regional de Psicologia sob a supervisão do professor orientador desta pesquisa. Depois de aplicados os inventários, serão realizadas a análise e a mensuração dos dados. Em uma perspectiva quantitativa pretende-se quantificar o número de estudantes com depressão, com ansiedade, com desesperança e suas escalas de intensidade.

A análise qualitativa assumirá o enfoque da Análise de Conteúdo proposta por Bardin. Os testes após avaliados irão fornecer resultados que subsidiarão entrevistas devolutivas a cada participante a respeito de suas informações. As entrevistas devolutivas serão realizadas pelo pesquisador de acordo com as normas e orientações do Conselho Regional de Psicologia sob a supervisão do professor orientador desta pesquisa.

\section{FORMA DE TRATAMENTO DOS DADOS E DE ANÁLISE E INTERPRETAÇÃO}

A análise quantitativa buscará realizar um levantamento dos dados coletados com base em amplitude total e ainda cruzando dados em relação aos diferentes sintomas e alterações de humos, assim como os entraves identificados no processo de ensino-aprendizagem.

A análise qualitativa será abordada sobre a perspectiva da Análise de Conteúdo de Bardin. Segundo Bardin (2011), fundamenta-se obter procedimentos sistemáticos, objetivos de descrições de conteúdos e indicadores se assim for, trazer para o centro das discussões e, consequentemente o embate dessa dialética, sem a preocupação do resultado ser positivo ou negativo.

De acordo com Godoy (1995) a pesquisa qualitativa não procura enumerar ou medir os eventos estudados:

Parte de questões ou focos de interesses amplos, que vão se definindo a Análise de conteúdo: da teoria à prática em pesquisas sociais aplicadas as organizações à medida que o estudo se desenvolve. Envolve a obtenção de dados descritivos sobre pessoas, lugares e processos interativos pelo contato direto do pesquisador com a situação estudada, procurando compreender os fenômenos segundo a perspectiva dos sujeitos, ou seja, dos participantes da situação em estudo (GODOY, 1995, p.58).

\section{SOBRE OS RESULTADOS}

Os resultados poderão subsidiar novas pesquisas e aprofundar pesquisas já existentes acerca da condição emocional de estudantes universitários. Podendo ainda, de maneira preliminar, oferecer informações, para trabalhos acadêmicos que busquem identificar ou se empenhar no desenvolvimento de práticas de bons cuidados em relação à qualidade de vida destes estudantes. Oferecer dados que contribuam para a compreensão das demandas em saúde mental da população estudada. Os resultados serão apresentados oportunamente e pelo menos um artigo será elaborado a partir da análise e conclusão do trabalho em questão.

\section{REFERÊNCIAS}

ASSOCIAÇÃO AMERICANA DE PSIQUIATRIA. Manual diagnóstico e estatístico de transtornos mentais: DSM-IV®. 4. ed. Porto Alegre, RS: Artes Médicas, 1995. 
ASSOCIAÇÃO AMERICANA DE PSIQUIATRIA. Manual diagnóstico e estatístico de transtornos mentais: DSM-V®. Porto Alegre, RS: Artes Médicas, 2014.

BARDIN, L. Análise de conteúdo. São Paulo: Edições 70, 2011.

BECK, A. T.; STEER, R. A. Beck Anxiety Inventory. Manual. San Antonio, TX: Psychological Corporation, 1993.

BECK, J. S. Terapia cognitiva: teoria e prática. Tradução Sandra Costa. Porto Alegre: Artmed, 1997.

BRASIL. Ministério da Educação e Cultura. Portaria Nº 343, de 17 de março de 2020. Dispõe sobre a substituição das aulas presenciais por aulas em meios digitais enquanto durar a situação de pandemia do Novo Coronavírus COVID-19. Diário Oficial da União, publicado em: 18/03/2020, Ed.53, seção 1, p. 39. Disponível em : <http://www. in.gov.br/en/web/dou/-/portaria-n-343-de-17-de-marco-de-2020-248564376>. Acesso em: 15 mar. 2020.

BRASIL. Ministério da Saúde. Uma análise da situação de saúde. Brasília: MS, 2007. Disponível em: <http://bvsms.saude.gov.br/bvs/publicacoes/saude_brasil_2007.pdf>. Acesso em: 10 abr. 2020.

CUNHA, Jurema Alcides. Manual em português das Escalas Beck / Jurema Alcides Cunha. São Paulo: Casa do Psicólogo, 2001.

DALGALARRONDO P. Psicopatologia e semiologia dos transtornos mentais. Porto Alegre: Artes Médicas; 2000.

DAMÁSIO, B. Mensurando habilidades socioemocionais de crianças e adolescentes: desenvolvimento e validação de uma bateria. (Nota técnica). Bruno Figueiredo Magalhães. Rio de Janeiro: Departamento de Psicometria da Universidade Federal do Rio de Janeiro, 2017.

FIOCRUZ, Fundação Oswaldo Cruz: uma instituição a serviços da vida. Disponível em: <https://portal.fiocruz.br/ pergunta/por-que-doenca-causada-pelo-novo-virus-recebeu-o-nome-de-covid-19>. Acesso em: 01 maio 2020.

GODOY A. S. Introdução à pesquisa qualitativa e suas possibilidades. RAE-Revista de Administração de Empresas, v. 35, n. 2, mar-abr, p. 57-63, 1995.

GORENSTEIN, C.; WANG, Y.; ARGIMON, I. I. D. L.; WERLANG, B. S. G. BDI-II: Inventário de Depressão de Beck II / Adaptação para o português. São Paulo: Casa do Psicólogo, 2011.

SPINK, M. J. Trópicos do discurso sobre risco: risco-aventura como metáfora na modernidade tardia. Cad. Saúde Pública, Rio de Janeiro, v. 17, n. 6, p.1277-1311, nov-dez, 2001. 